\title{
EINIGE BEZEICHNUNGEN
}

Verteilungsfunktion der Teilchen $f$ (Kapitel I-VI); in den Impulsen immer auf $\mathrm{d}^{3} p$ bezogen.

Verteilungsfunktion als Besetzungszahl quantenmechanischer Zustände der Elektronen und Phononen $n(\boldsymbol{p})$ und $N(\boldsymbol{k})$ (Kapitel VII, IX-XI); in den Impulsen immer auf $\mathrm{d}^{3} p /(2 \pi \hbar)^{3}$ bezogen.

Stoßintegral St, linearisiertes Stoßintegral $I$.

Thermodynamische Größen: Temperatur $T$, Druck $P$, chemisches Potential $\mu$, Teilchenzahldichte $N$, Gesamtteilchenzahl $\mathscr{N}$, Gesamtvolumen $v$.

Elektrische Feldstärke $\boldsymbol{E}$, magnetische Induktion $\boldsymbol{B}$. Elektrische Elementarladung $e$ (Ladung des Elektrons -e).

Bei Abschätzungen werden folgende Bezeichnungen benutzt: charakteristische Längen des Problems $L$; atomare Abmessungen, Gitterkonstante $d$; freie Weglänge $l$; Schallgeschwindigkeit $u$.

Die Mittelung wird durch spitze Klammern $\langle\ldots\rangle$ oder durch Utberstreichen des Buchstaben gekennzeichnet.

Dreidimensionale Vektorindizes werden durch griechische Buchstaben $\alpha, \beta, \ldots$ bezeichnet.

In den Kapiteln III-VI:

Elektronenmasse $m$, Ionenmasse $\boldsymbol{M}$.

Elektronenladung $-e$, Ionenladung $z e$.

Thermische Geschwindigkeiten der Elektronen und Ionen

$$
v_{T e}=\left(T_{e} / m\right)^{1 / 2}, \quad v_{T i}=\left(T_{i} / M\right)^{1 / 2} .
$$

Plasmafrequenz

$$
\Omega_{e}=\left(4 \pi N_{e} e^{2} / m\right)^{1 / 2}, \quad \Omega_{i}=\left(4 \pi N_{i} z^{2} e^{2} / M\right)^{1 / 2} .
$$

DEB Ye-Radius

$$
a_{e}=\left(T_{e} / 4 \pi N_{e} e^{2}\right)^{1 / 2}, \quad a_{i}=\left(T_{\mathfrak{i}} / 4 \pi N_{i} z^{2} e^{2}\right)^{1 / 2}, \quad a^{-2}=a_{e}^{-2}+a_{i}^{-2} .
$$

Zyklotronfrequenz

$$
\omega_{B e}=e B / m c, \quad \omega_{B i}=z e B / M c .
$$


Die Hinweise auf Paragraphennummern und Formeln anderer Bände dieses Lehrwerkes sind mit römischen Ziffern versehen:

I - Mechanik, 1981; II - Klassische Feldtheorie, 1981; III - Quantenmechanik, 1979; IV - Relativistische Quantentheorie, 1980; V - Statistische Physik, Teil 1, 1979; VI - Hydrodynamik, 1978; VII - Elastizitätstheorie, 1975; VIII - Elektrodynamik der Kontinua, 1980; IX - Statistische Physik, Teil 2, 1980. 